\title{
Is Early Compression Therapy after Perforator Flap Safe and Reliable?
}

\author{
Hyunsuk Peter Suh, MD, PhD ${ }^{1}$ Hyung Hwa Jeong, $\mathrm{MD}^{1}$ Joon Pio (Jp) Hong, MD PhD, MMM ${ }^{1}$ \\ 1 Department of Plastic and Reconstructive Surgery, Asan Medical \\ Center, University of Ulsan College of Medicine, Seoul, Korea \\ J Reconstr Microsurg 2019;35:354-361.

\begin{abstract}
Address for correspondence Joon Pio (Jp) Hong, MD, PhD, MMM, Center, University of Ulsan College of Medicine, 88 Olympic-ro 43-gil, Songpa-gu, Seoul 05505, Korea (e-mail: joonphong@amc.seoul.kr).
\end{abstract} \\ Department of Plastic and Reconstructive Surgery, Asan Medical
}

\begin{abstract}
Keywords

- perforator flap

- compression of flaps

- flap surgery

Background Due to the concern of circulation compromise, many surgeons hesitate to dangle or challenge the flap in the early postoperative phase. In our experience, early compression may have advantages such as collapse of dead space, evacuation of hematoma, reduction of edema, and stabilization of flap when ambulating. In this study, the effect of early compression of flaps with regard to flap physiology and outcome is evaluated.

Methods From postoperative day 3 to 5 , a custom-made compression garment was fitted aiming to compress with a pressure of 30 to $35 \mathrm{mmHg}$. A total of 52 patients (52 flaps) were enrolled for the study and 48 flaps underwent early compression. The compression was applied not only over the flap but also over the extremity or trunk. The flap outcome as well as flow velocity and flow volume of the pedicle before and after compression was measured and evaluated.

Results There was no flap complication during the early compression and early ambulation. And, there was no statistical difference either in the flow velocity or in the volume before and after the compression therapy $(29.21$ vs. $29.42 \mathrm{~cm} / \mathrm{s}$ and 7.13 vs. $6.56 \mathrm{~cm}^{3} / \mathrm{s}$, respectively).

Conclusion Early compression with a $30-\mathrm{mmHg}$ pressure customized pressure garment after the flap surgery does not affect the hemodynamics of the perforator and can be safely used after extremity reconstruction before ambulation.
\end{abstract}

When a patient starts ambulation after extremity reconstruction, limb swelling, transient congestion, and edema are commonly caused by gravity and mobilization resulting in hydrostatic pressure putting a strain on the flaps. ${ }^{1}$ When the flap is especially on the weight-bearing area, the hydrostatic pressure, venous congestion, and mechanical tension on the flap or flap margin can also cause wound dehiscence. Thus, challenging flaps before starting ambulation has been investigated in several studies. Some studies were especially conducted to reveal the microcirculatory basis of the dangling procedure. Kolbenschlag et al revealed that after the dangling procedure the oxygenation and hemoglobin concentration in the flap increased. ${ }^{2}$ Although the flap adjust-

received

July 26, 2018

accepted after revision

October 18, 2018

published online

December 17, 2018 ment such as dangling is clearly needed, the timing of dangling/wrapping procedures remains controversial. Some studies advocate early dangling application, while other studies argue the need for latent application. ${ }^{3-5}$ However, there is a trend toward early dangling and wrapping after flap surgery. Nevertheless, surgeons still hesitate to start dangling, wrapping, and early ambulation after free flap reconstruction due to the concern of circulatory compromise.

In our practice, we have been using early compression of flaps, especially skin flaps, and found that it benefits the overall outcome as well as the early phase of the flap. To further support our experience, we measured the
Copyright $\odot 2019$ by Thieme Medical Publishers, Inc., 333 Seventh Avenue, New York, NY 10001, USA. Tel: +1(212) 584-4662.
DOI https://doi.org/ 10.1055/s-0038-1676604. ISSN 0743-684X. 
compression pressure in our postoperative compression protocol and studied the flow of the perforator pedicle to see whether any vascular compromise exists under the flap after the compression. We hypothesized that early compression of 30 to $35 \mathrm{mmHg}$ is safe and can benefit the flap. The goal of this study was to evaluate the effect of early flap compression by studying the flow velocity and flow volume during the compression.

The benefits for early flap challenges such as dangling and bandage compression are still controversial. Furthermore, although some challenging procedures for flaps have been documented in several studies, reports documenting the exact timing or protocol of flap compression in the early period as a challenging procedure before engaging flaps to the new environment are rare. ${ }^{6}$

\section{Methods}

\section{Patients}

From December 2016 to August 2017, 52 patients who had perforator-based flaps on the extremity and trunk, such as propeller flaps and free flaps, and agreed to measure the pressure were enrolled in this study. This study was reviewed by the Institutional Review Board at the Asan Medical Center.

\section{Compression Protocol}

In our institution, we routinely apply the early compression protocol (from postoperative day 3 to 5 ) to all flaps when applicable. Flaps that are used for resurfacing can be candidates for compression with the exception of flaps that are used for an ischemic limb. Prior to the reconstruction, the garments are usually designed based on the mirror measurements of the contralateral limb with the aim of generating a compression pressure of 30 to $40 \mathrm{mmHg}$. When a mirror image is not possible such as the torso, the compression garment is tailored after manual measurement and then fitted accordingly to reach the target pressure. As the flap swelling is reduced and molded into contour, the garment can be adjusted accordingly. The area covered by the compression garment includes the microanastomosis site and the flap pedicle (- Fig. 1, left and center).

Prior to full compression, a mild compression is performed that usually lasts 1 or 2 days starting from postoperative day 3 or 4 . The flap is wrapped with a soft, cohesive, air permeable, elastic-conforming bandage (Pehahaft, Paul Hartmann AG, Heidenheim, Germany). If the flap endures the mild compression, custom-made compression garments with 30 to $40 \mathrm{~mm} \mathrm{Hg}$ pressure are applied on day 5. A thin foam dressing is used to cover the flap under the
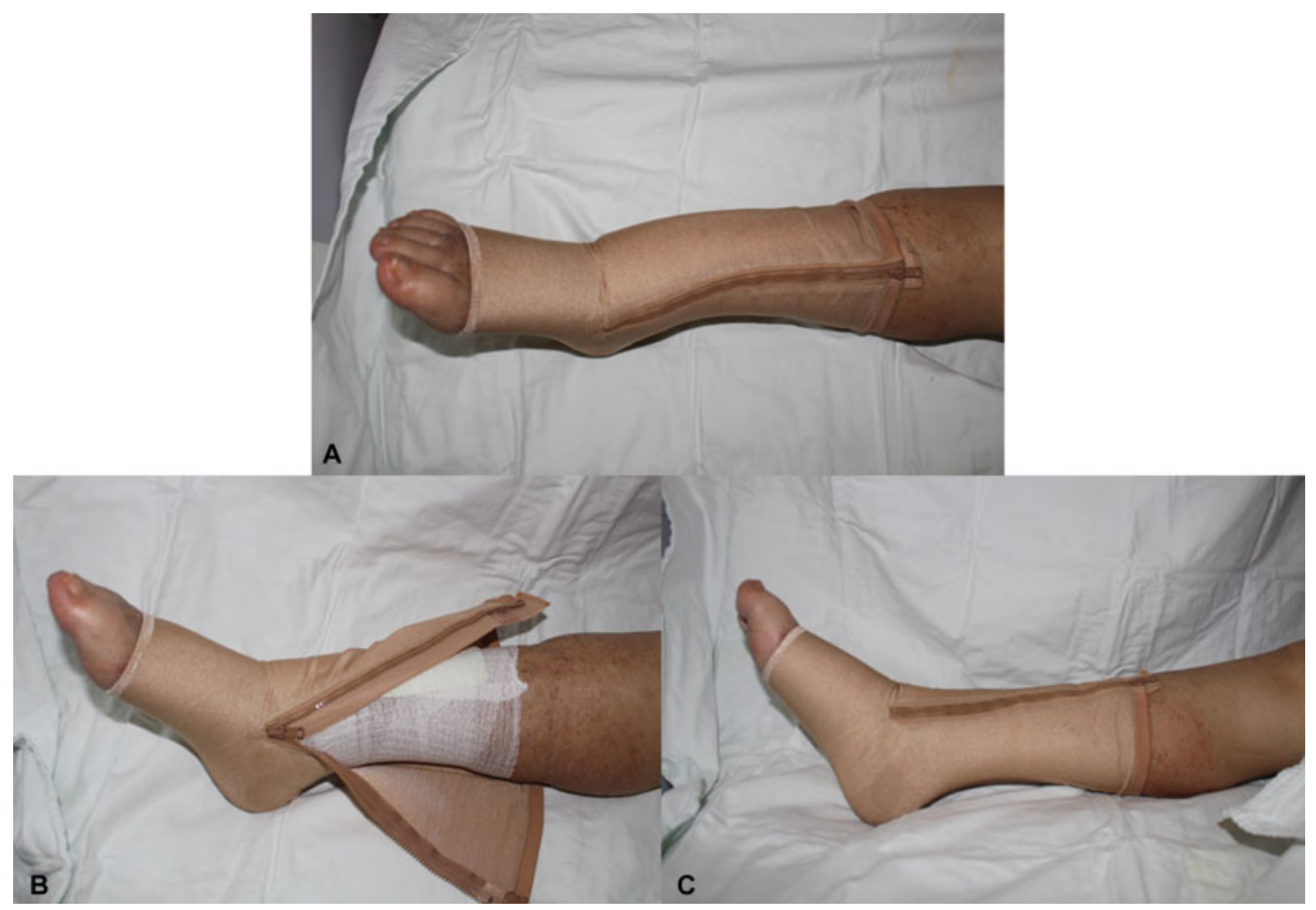

Fig. 1 Application of custom-made compression garment is shown after reconstructing the lateral malleolus defect of right foot (A). The area covered by the garment includes the area of microanastomosis, the entire flap, and the surrounding tissue (B). Note the first layer with foam dressing and mild bandage before compression which is done for 2 weeks during the early healing phase (C). After the dressing, the compression is zipped up. 
compression garment (-Fig. 1, right). The compression starts from 2-hour duration for three times per day gradually increasing to 6-hour duration for three times per day ultimately reaching continuous compression usually by the sixth day of application. During the compression, if flap refill shows a sudden change, compression is stopped and managed accordingly.

Early compression must be avoided when flaps show venous congestion or suspicion of flap ischemia. The application may be postponed till the problem has resolved. Padding with foam dressing can be useful over bony prominence as the compression may result in pressure sores.

\section{Measuring Compression Pressure, Flap Flow Velocity, and Flow Volume}

First, the flow velocity and volume of the flap are measured before compression using a duplex ultrasound (LOGIQ E9/GE, GE Healthcare, Milwaukee, WI). Then, the compression is applied and the pressure is measured using a pressure measuring device (Palm Q CR-490, Cape, Japan) to ensure optimal pressure. Because of the bulkiness of the probe as it may affect the measurement by increasing the local pressure, we had to design a model where the probe pressure does not affect the measurements. The flap was compressed using an elastic bandage with the same compression pressure as the compression garment (-Fig. 2). Then a small opening

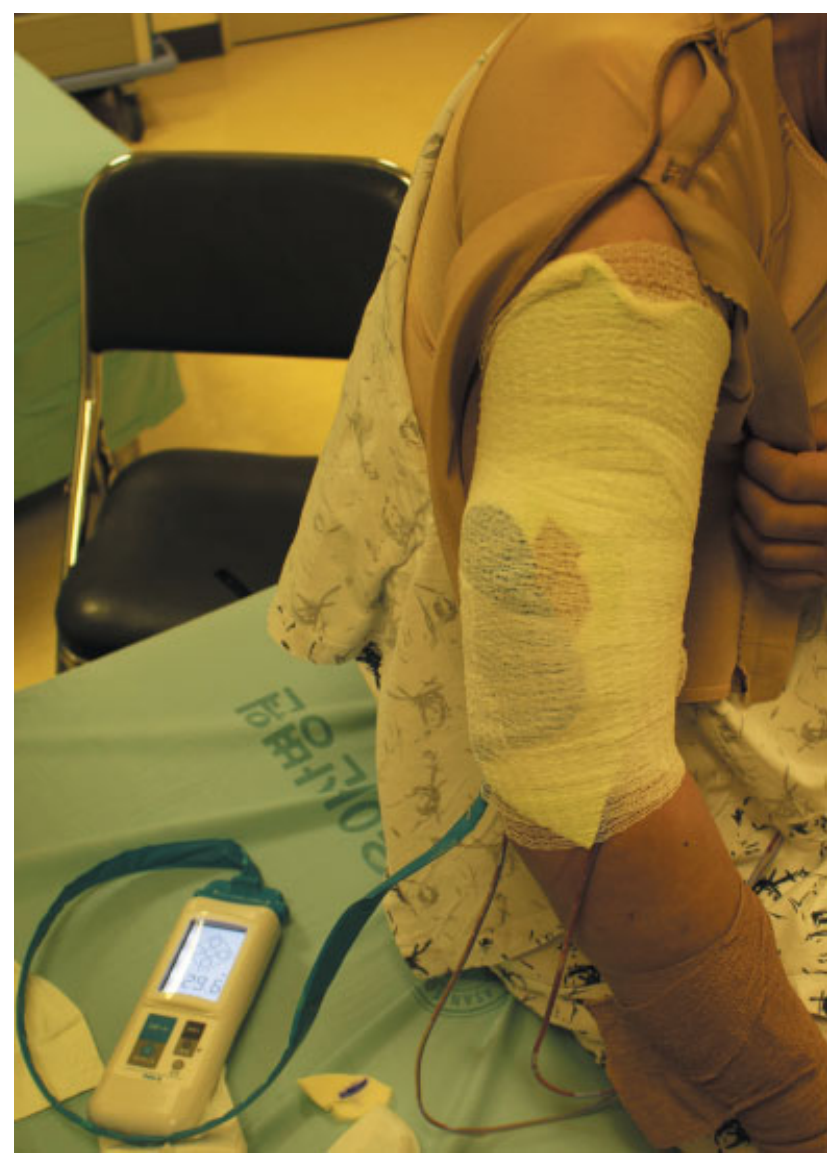

Fig. 2 Measurement of the pressure of compression garment with a pressure sensor. The identical pressure was also simulated with an elastic bandage.
$(<2 \mathrm{~cm})$ on the dressing was made to allow the probe to measure the flow velocity and flow volume of the perforator site. The paired $t$-test was used to compare the pressure between the compression garment and the elastic bandage.

Second, the flow velocity and flow volume of the flap pedicle are measured before and after the compression therapy. The paired $t$-test was used to compare the flow velocity and flow volume prior and after application of compression.

Finally, the flow velocity and flow volume were measured in respect to the anastomosis style. The repeated measures analysis of variance (ANOVA) test was used to compare the data.

All statistics used SSPS (IBM SPSS Statistics for Windows, Version 18.0, IBM Corp. Armonk, NY).

\section{Ambulation Protocols}

For lower extremity reconstruction, the patients are allowed to ambulate at day 5 under compression. For nonweight bearing flaps, full-weight bearing was commenced at 7 to 14 days if tolerable. If there was skeletal instability, ambulation was initiated accordingly. For weight-bearing flaps, compression was applied on day 5 along with short leg splints and partial-weight bearing began with the aid of walkers, gradually reaching full weight bearing at 3 to 6 weeks when the splints were removed. Usually the patient is discharged at day 7 to 10 educated with the protocol and visits the outpatient clinic every week for follow-up.

For other regions, ambulation began whenever tolerable but the compression started from day 5 with the same protocol.

The weaning of the compression garment usually ensues after several months. If the flap does not swell without applying the garment after a full day of activity, then the patient can stop using compression. As for weight-bearing flaps, compression is recommended whenever the patient has to gait for a long time to minimize any shearing.

\section{Results}

The overall characteristics of the patients are listed in - Table 1. Among 52 flaps in 52 patients, early compression was not initiated in 4 patients because of flap congestion. The average postoperative day for measuring compression pressure and the flow was at day 5 (-Table 2). The average follow-up of the patients was 15 months (range: 11-26 months).

There was no wound dehiscence after compression in all 48 patients with early application. Neither flap necrosis nor other major complication such as bleeding that requires a revisionary operation occurred after early compression.

The average pressure of the compression garment was $29.56 \mathrm{mmHg}$. The average pressure reproduced with the compressive bandage for measurement was $27.81 \mathrm{mmHg}$ (-Table 2). There was no significant difference between the compression garment pressure and reproduced pressure $(p=0.128)$. 
Table 1 Baseline statistics of the flaps

\begin{tabular}{|l|l|}
\hline Flap & N $=\mathbf{5 2}$ \\
\hline SCIP free flap & 26 \\
\hline ALT free flap & 15 \\
\hline PAP free flap & 1 \\
\hline SIEA free flap & 1 \\
\hline Propeller flap & 8 \\
\hline Miscellaneous $^{\text {a }}$ & 1 \\
\hline Anastomosis & \\
\hline End-to-end & 14 \\
\hline End-to-side & 27 \\
\hline Flow through & 2 \\
\hline Location $^{\text {to }}$ & \\
\hline Foot & 19 \\
\hline Knee & 5 \\
\hline Leg $^{2}$ & 17 \\
\hline Thigh & 5 \\
\hline Upper extremity & \\
\hline Trunk & 2 \\
\hline
\end{tabular}

Abbreviations: ALT, anterolateral thigh; PAP, profunda femoris artery perforator; SCIP, superficial circumflex iliac artery perforator; SIEA, superficial inferior epigastric artery.

apedicled ALT flap.

${ }^{\mathrm{b}}$ Only in free-flap cases $(n=43) .^{\mathrm{c}}$ Wrist, upper arm.

Table 2 Summary of compression statistics

\begin{tabular}{|l|l|}
\hline & (Total, $\boldsymbol{n}=\mathbf{5 2}$ ) \\
\hline $\begin{array}{l}\text { Average pressure of } \\
\text { compressive garment }\end{array}$ & $29.56 \mathrm{mmHg}$ \\
\hline $\begin{array}{l}\text { Average reproduced } \\
\text { pressure with bandage }\end{array}$ & $27.81 \mathrm{mmHg}$ \\
\hline
\end{tabular}

The average velocity of the pedicle of all flaps enrolled to the study before compression was $29.21 \mathrm{~cm} / \mathrm{s}$. After initiating compression treatment with a custom-made compression
Table 3 Change of flap perfusion velocity and volume after compression (compared using student $t$-test)

\begin{tabular}{|c|c|c|}
\hline \multicolumn{3}{|l|}{ Average velocity } \\
\hline Before compression & $29.21 \mathrm{~cm} / \mathrm{s}$ & \multirow[t]{2}{*}{$p=0.880$} \\
\hline After compression & $29.42 \mathrm{~cm} / \mathrm{s}$ & \\
\hline \multicolumn{3}{|l|}{ Average volume } \\
\hline Before compression & $7.13 \mathrm{~cm}^{3} / \mathrm{s}$ & \multirow[t]{2}{*}{$p=0.158$} \\
\hline After compression & $6.56 \mathrm{~cm}^{3} / \mathrm{s}$ & \\
\hline
\end{tabular}

garment or an elastic bandage, the average velocity of the flap pedicle was measured to be $29.42 \mathrm{~cm} / \mathrm{s}$. The average pedicle perfusion volume was also calculated before and after compression treatment $\left(7.13 \mathrm{~cm}^{3} / \mathrm{s}\right.$ and $6.56 \mathrm{~cm}^{3} / \mathrm{s}$, respectively). There was no statistical difference in flow velocity or flow volume at the pedicle of the flap before and after the compression therapy (-Table $\mathbf{3}$ ). In addition, we compared the mean velocity and perfusion volume with regard to the pedicle anastomosis type. There were no significant difference in velocity and volume after the compression therapy with regard to the various pedicle anastomosis methods in free flaps (-Tables 4 and 5). All flaps survived without major complication of flap loss.

-Figs. 3, 4, and 5 depict typical cases and approaches of compression on flaps.

\section{Discussion}

However, the definition of compression with regard to the amount of pressure and frequency was not clear. The findings from this study present a detailed application along with a reliable safety margin formulating an "early aggressive flap compression model."

The first question that is raised when considering compression is the safety regarding how much pressure to use. The pressure of 30 to $35 \mathrm{mmHg}$ was determined based on the fact that compression stocking to minimize and prevent orthostatic swelling uses 20 to $30 \mathrm{mmHg}$. We further determined the safety of compression by looking at the survival of

Table 4 Comparison of velocity change between pedicle anastomosis types in free flaps

\begin{tabular}{|l|l|l|l|l|l|}
\hline \multicolumn{2}{|l|}{ Total $=43$} & Standard error & \multirow{2}{*}{$p$-Value } & \multicolumn{2}{l|}{ 95\% Confidence group } \\
\cline { 3 - 6 } & & & Lower bound & Upper bound \\
\hline \multirow{2}{*}{ End-to-side $(n=27)$} & End-to-end & 2.22257 & 0.780 & -6.9084 & 3.9107 \\
\cline { 2 - 6 } & Flow through & 4.94552 & 0.995 & -11.5680 & 12.5060 \\
\hline \multirow{2}{*}{ End-to-end $(n=14)$} & End-to-side & 2.22257 & 0.780 & -3.9107 & 6.9084 \\
\cline { 2 - 6 } & Flow through & 5.10142 & 0.921 & -10.4486 & 14.3843 \\
\hline Flow through $(n=2)$ & End-to-side & 4.94552 & 0.995 & -12.5060 & 11.5680 \\
\cline { 2 - 6 } & End-to-end & 5.10142 & 0.921 & -14.3843 & 10.4486 \\
\hline
\end{tabular}

Note: Repeated measures analysis of variance (ANOVA) test was used. 
Table 5 Comparison of volume change between pedicle anastomosis types in free flaps

\begin{tabular}{|l|l|l|l|l|l|}
\hline \multicolumn{2}{|l|}{ Total $=43$} & Standard error & \multirow{2}{*}{-Value } & \multicolumn{2}{l|}{ 95\% Confidence group } \\
\cline { 3 - 6 } & & & \multicolumn{2}{l|}{ Lower bound } & Upper bound \\
\hline \multirow{2}{*}{ End-to-side $(n=27)$} & End-to-end & 1.76162 & 0.599 & -2.5760 & 5.9993 \\
\cline { 2 - 6 } & Flow through & 3.91985 & 0.578 & -5.6018 & 13.4794 \\
\hline \multirow{2}{*}{ End-to-end $(n=14)$} & End-to-side & 1.76162 & 0.599 & -5.9993 & 2.5760 \\
\cline { 2 - 6 } & Flow through & 4.04341 & 0.847 & -7.6142 & 12.0685 \\
\hline Flow through $(n=2)$ & End-to-side & 3.91985 & 0.578 & -13.4794 & 5.6018 \\
\cline { 2 - 6 } & End-to-end & 4.04341 & 0.847 & -12.0685 & 7.6142 \\
\hline
\end{tabular}

Note: Repeated measures analysis of variance (ANOVA) test was used.

the flap as well as the effect on the pedicle flow velocity and volume. Our results at 30 to $35 \mathrm{mmHg}$ compression on flaps show no difference in flow velocity of the pedicle prior $(29.21 \mathrm{~cm} / \mathrm{s})$ and post $(29.42 \mathrm{~cm} / \mathrm{s})$ compression. The same can be seen for the flow volume prior $7.13\left(\mathrm{~cm}^{3} / \mathrm{s}\right)$ and post $\left(6.56 \mathrm{~cm}^{3} / \mathrm{s}\right)$ compression. Despite the trend that end-to-end anastomosis leads to higher pressure and stronger flow, the compression despite the different anastomosis styles had no significant effect either on the velocity or on the flow volume. The reason for starting at day 3 to 5 was to allow the flap to inosculate to the surrounding structure and to monitor the flap for possible complications. ${ }^{7}$ Thus the implemented compression protocol did not exert any significant change in flow velocity or volume leading to no major complication during the follow-up.

Our experience showed that the use of custom-designed pressure garments had many benefits. Measurement was made directly over the reconstructed extremity or in cases where direct measurement was difficult, such as patients using external fixators, mirroring measurements were used

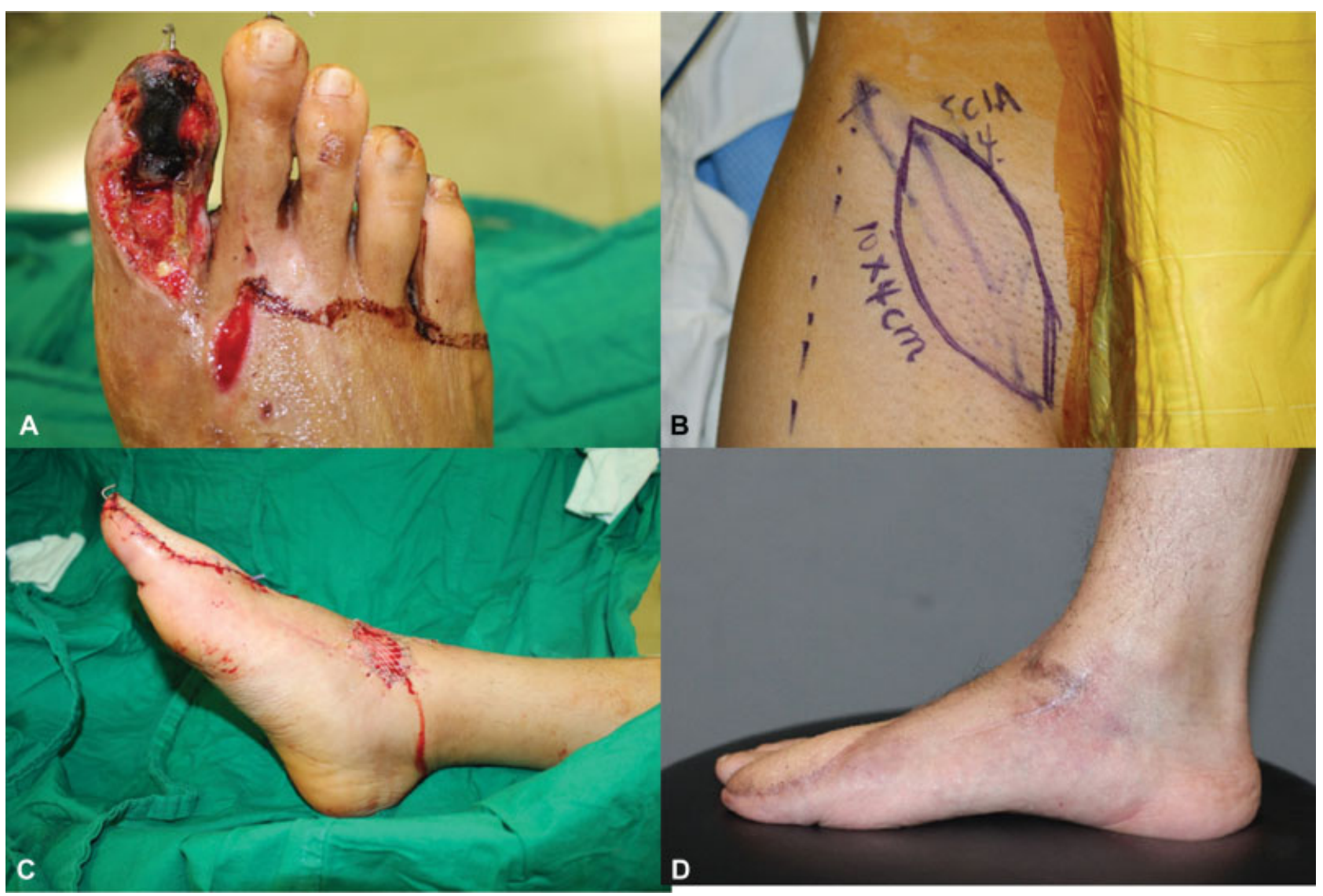

Fig. 3 A 31-year-old man is presented with a defect caused by crushing injury of the right first toe (A). After debridement, a superficial circumflex iliac artery perforator free flap was elevated. The artery was anastomosed to the digital artery of the first toe in an end-to-end manner and the vein was anastomosed to the superficial vein in an end-to-end manner (B). Postoperative image is shown prior to compression (C). As the flap was stable, early compression from postoperative day 5 was performed according to the protocol with the compression garment applied over the entire foot. Note the findings at 7 months after reconstruction which shows good contour (D). 


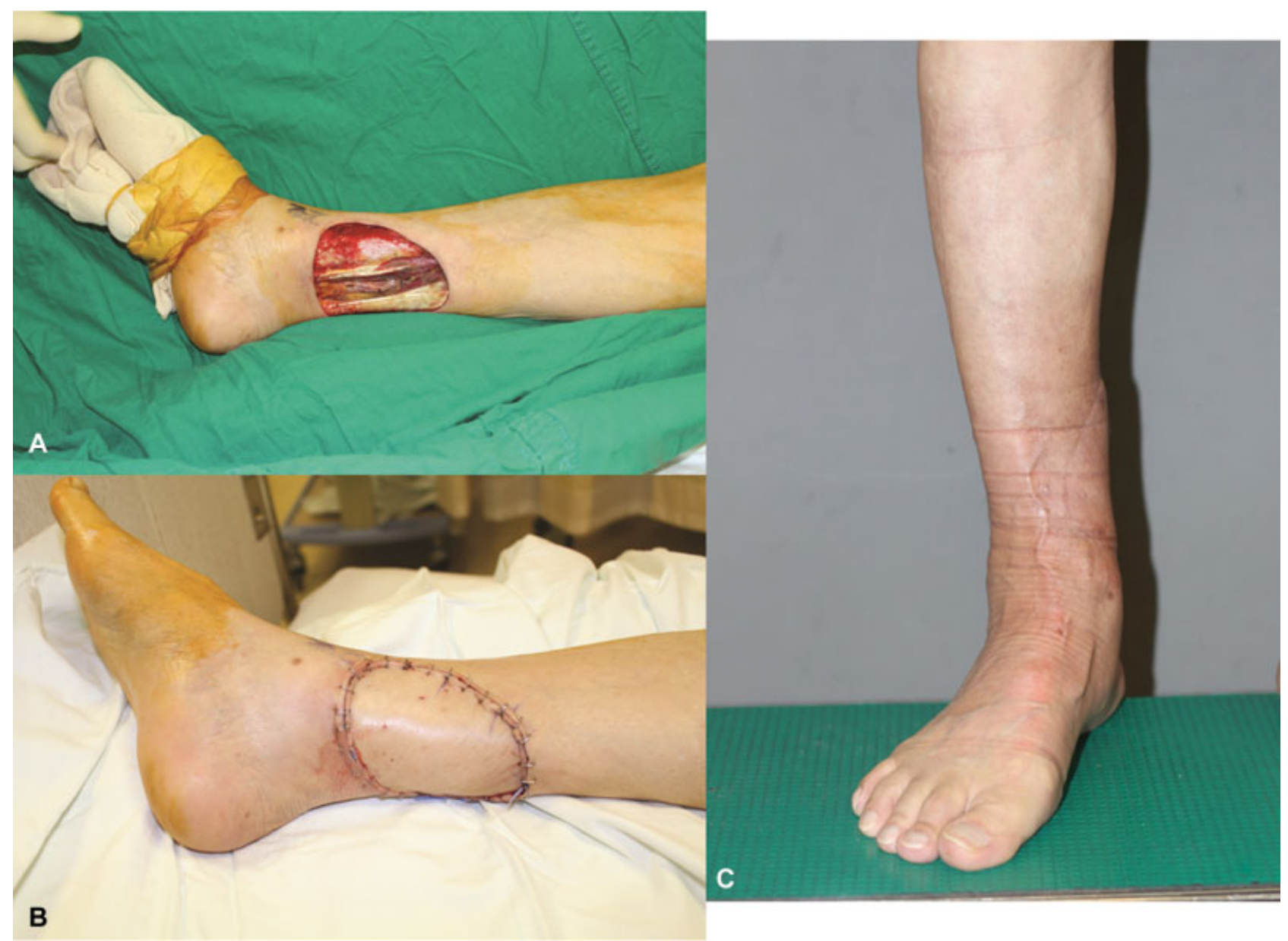

Fig. 4 A 76-year-old man is presented with undifferentiated pleomorphic sarcoma on his right medial ankle. After wide excision, a $12 \times 8 \mathrm{~cm}$ sized defect is shown (A). A superficial iliac artery perforator free flap was elevated and the artery was anastomosed to the posterior tibial artery in an end-to-side manner along with two veins anastomosed to two accompanying veins in an end-to-end manner. The flap with moderate swelling prior to compression is noted (B). The early compression protocol was applied starting from postoperative day 5 and started partial ambulation. The patient was discharged on postoperative day 7 with a stable flap under compression and full weight bearing ambulation. The patient was still under full time compression at 2 months showing good contour of the flap (C).

and then tailored accordingly. The pressure garments bring constant and even pressure over the extremity, while the wrapping procedure may vary depending on the caregiver. This garment is not new and is frequently used in burn patients to minimize scar formation. It is easily available, has a durable texture, and can be tailored to reduce or expand the fabric to custom fit the extremity or trunk according to the progress of the flap. Since the garment can be utilized for a long period, there are cost benefits as well. Finally, with a zipper application over the protection fabric is safe to use and easy to close without any aid.

The second question frequently asked is how does compression work? To answer this question, one should understand the principle for dangling. What are the effects of dangling? Jokuszies et al summarized the effect of the dangling procedure by venous drainage support, alleviation of the edema-associated problems. ${ }^{3}$ Jokuszies et al explained that the alleviation of edema-associated problems is related to the veno-arterial reflex mechanism. Studies have demonstrated that the human body can limit lower extremity edema by decreasing blood flow when venous pressure is elevated. Vascular resistances increase within a limb when venous pressure is higher than $25 \mathrm{mmHg}$, which leads to limitation of the inflow. ${ }^{8}$ Also during initial dangling, the oxygenation level elevates initially but drops to the nadir for several minutes and gradually recovers. ${ }^{9}$ Thus conditioning by dangling allows the flap to adjust to venous pooling without impairing oxygenation and avoid other complications. ${ }^{10}$ However, without preparing the flap through dangling, peripheral hyperemia, congestion, altered perfusion, and increased flow to the lower extremity can occur leading to edema both at the lower limb and at the transferred flap. $^{3,11}$ Edema impedes revascularization and causes delayed wound healing. Thus we hypothesized that minimizing edema may reduce venous pooling, omitting the need for dangling and thus leading to early ambulation. Although we were unable to document the change in venous flow, it was most likely that the compressed veins underwent a similar change as the arteries. In addition, we expected the compression on the flaps will exert pressure over the vein and reduce venous pooling and thus reduction of edema. The same principle of compression is used in venous ulcers and 


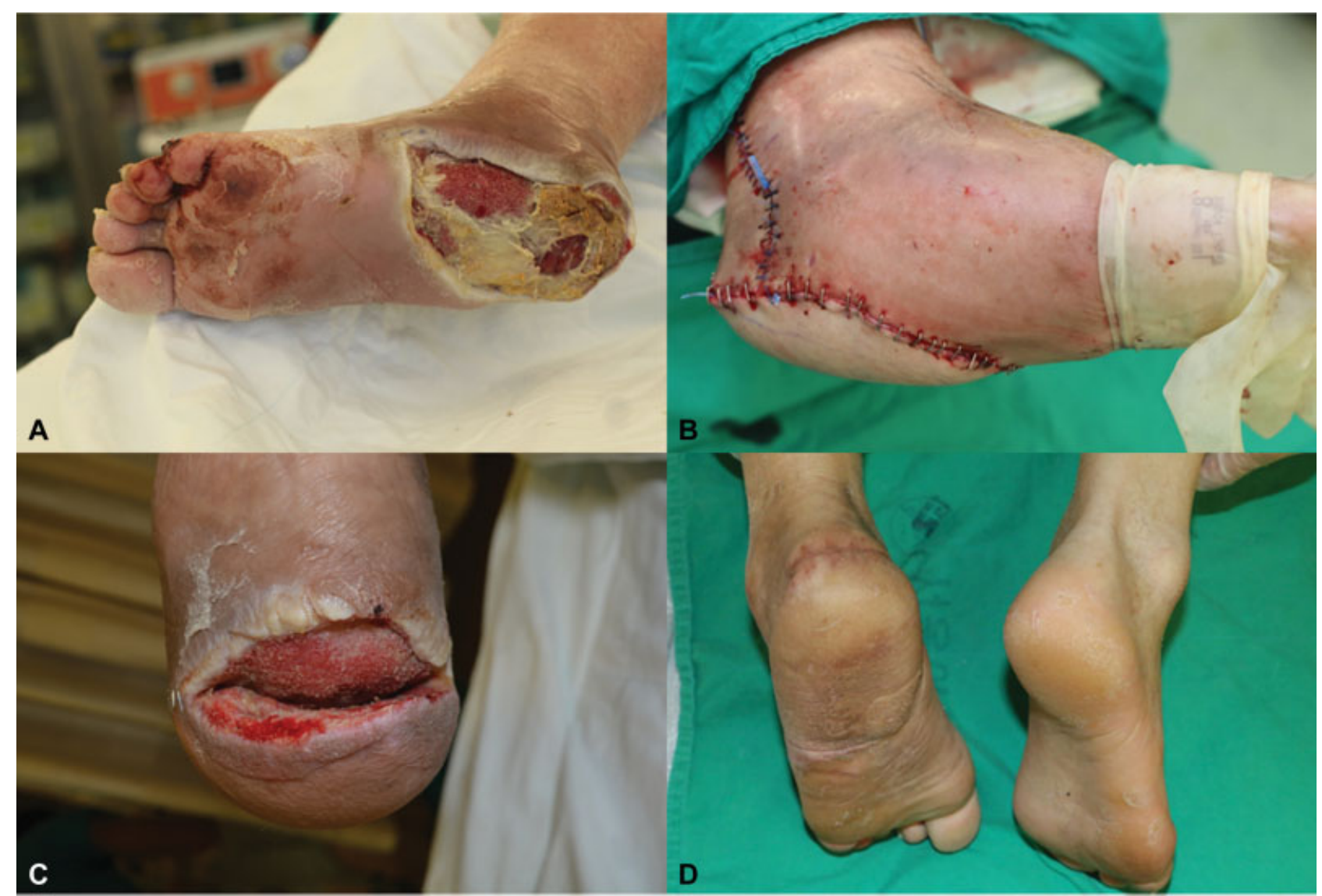

Fig. 5 A 53-year-old man underwent reconstruction of diabetic foot with Charcot foot deformity is presented. After multiple debridements, the defect shows clean but exposed tendon and bone with granulation (A). A $22 \times 9 \mathrm{~cm}$ anterolateral thigh free flap was designed and elevated from the right thigh. Vascular anastomosis was performed in an end-to-side manner for arterial anastomosis to the posterior tibial artery. Venous anastomosis was performed in an end-to-end manner for one accompanying vein. The early stage of reconstruction shows well-taken flap (B). The patient started wheelchair ambulation on postoperative day 5 and started compression with a custom-made compression garment from postoperative day 7 due to delayed healing by diabetes. The patient discharged uneventfully under compression and partial weight bearing at 2 weeks. However, the patient visited the clinic with posterior margin dehiscence at 1 month during follow-up (C). The patient was not compliant to compression garment application. After debridement and repair, the patient complied with the compression protocol and shows wellmaintained flap without further complication at 18 months (D).

lymphedema to improve the venous return and venolymphatic function. ${ }^{5}$ The significance of venous drainage in flap survival is documented in numerous studies. ${ }^{12-14}$ Thus our protocol with a compression garment can aid the flap recovery by avoiding venous pooling, maintaining a stable oxygenation level and supporting venous drainage. ${ }^{9}$

The third question is the timing of compression. Is early compression beneficial? Dangling as early as day 2 has shown to be safe without increasing complications and affords decreased hospital stays and early flap conditioning. ${ }^{10}$ Thus when the flaps is stable, early compression begins with less pressure and slowly builds the pressure up to 30 to $35 \mathrm{mmHg}$ by day 5 . As we have shown in our results, compression also stabilized the flap from shearing and led to early ambulation without the worry of venous pooling and to earlier discharge. An additional benefit of early compression is the physical compression effect. Some studies have demonstrated that dead space under the skin will result in seroma formation and seroma may lead to unwanted complications. ${ }^{15-17}$ Although the relationship between compression and seroma formation is controversial, we expect that the compression does have a role in reducing seroma-related complications by obliterating the dead space of the flap with appropriate pressure. ${ }^{18,19}$ In our experience, we note increased discharge from the flap margins when compression is applied early, supporting our notion.

Fourth, there is a physical and mechanical benefit of using compression especially for patients with reconstructed flaps on the foot. When patients begin ambulation on the reconstructed surface of the flap, the shearing force exerts at the flap, which may lead to dehiscence, scar formation, and ulceration of the flap. Constant movement and weight bearing endanger the flap to mechanical stress. We anticipated that the compression garment on the flap will decrease the tension on the margins of the flap, reduce shearing, and provide an extra layer of protection. Reduction of tension on the incision site has shown to be beneficial even just by using taping. ${ }^{20}$ Although a compression garment does not directly exert an effect on the margin as taping, it can minimize shearing by dynamic stretching and stabilization of the flap. ${ }^{21}$ Similar findings were noted for graft anchoring from the shearing force by using a two-way stretch net in 
pediatric $_{\text {patients. }}{ }^{22}$ The custom-made compression garment provides a four-way stretch that can produce individualized fitting covering around the flap. In one survey on reconstructive surgeons, plastic surgeons who answered to the survey agreed to start ambulation under a secure support with a compressive elastic bandage. ${ }^{5}$ We suspect that the compression garment serves as a barrier from the shearing force and provides mechanical support, especially in lower extremity reconstruction during ambulation. In our experience for plantar reconstruction, with aggressive education and continuous application of compression we have only had two minor cases of superficial ulceration.

Finally our experience shows that patients who have compression on their flaps have better overall contour. Prolonged edema plays a role in resulting in a bulky flap. With early compression, edema is better controlled resulting in better cosmetic appearance. In the age of thin flap elevation especially in perforator flaps, early compression to achieve better modelling of the flap can be meaningful.

\section{Conclusion}

Early compression with a 30 to $35 \mathrm{mmHg}$ customized pressure garment after flap surgery does not affect the hemodynamics of the perforator flap and can be safely used after reconstruction. The benefits include reduction of edema, obliteration of dead space, stabilization of flaps during ambulation, and achievement of good contour.

\section{Conflict of Interest}

None declared.

\section{References}

1 Cerny M, Schantz JT, Erne H, et al. Overview and introduction of a treatment concept for postoperative care and mobilisation after free flap transplantation in the lower extremity [in German]. Handchir Mikrochir Plast Chir 2016;48(06):363-369

2 Kolbenschlag J, Bredenbroeker P, Daigeler A, et al. Changes of oxygenation and hemoglobin-concentration in lower extremity free flaps during dangling. J Reconstr Microsurg 2014;30(05): 319-328

3 Jokuszies A, Neubert N, Herold C, Vogt PM. Early start of the dangling procedure in lower extremity free flap reconstruction does not affect the clinical outcome. J Reconstr Microsurg 2013;29 (01):27-32

4 Neubert N, Vogt PM, May M, et al. Does an early and aggressive combined wrapping and dangling procedure affect the clinical outcome of lower extremity free flaps? A randomized controlled prospective study using microdialysis monitoring. J Reconstr Microsurg 2016;32(04):262-270
5 Rohde C, Howell BW, Buncke GM, et al. A recommended protocol for the immediate postoperative care of lower extremity free-flap reconstructions. J Reconstr Microsurg 2009;25 (01):15-19

6 Soteropulos CE, Chen JT, Poore SO, Garland CB. Postoperative management of lower extremity free tissue transfer: a systematic review. J Reconstr Microsurg 2018

7 Lee CH, Han SK, Dhong ES, Kim HP, Kim WK. The fate of microanastomosed digital arteries after successful replantation. Plast Reconstr Surg 2005;116(03):805-810

8 Crandall CG, Shibasaki M, Yen TC. Evidence that the human cutaneous venoarteriolar response is not mediated by adrenergic mechanisms. J Physiol 2002;538(Pt 2):599-605

9 Ridgway EB, Kutz RH, Cooper JS, Guo L. New insight into an old paradigm: wrapping and dangling with lower-extremity free flaps. J Reconstr Microsurg 2010;26(08):559-566

10 Seth AK, Diamond S, Iorio ML. Outcomes of an early protocol for dependent conditioning in lower extremity microsurgical free flaps. J Reconstr Microsurg 2017;33(09):670-678

11 Dornseifer U, Kleeberger C, Kargl L, et al. Perfusion controlled mobilization after lower extremity free flaps-pushing the limits of time and intensity. J Reconstr Microsurg 2017;33(03): 179-185

12 Chow SP, Chen DZ, Gu YD. The significance of venous drainage in free flap transfer. Plast Reconstr Surg 1993;91(04):713-715

13 Fukui A, Tamai S, Williams HB. The importance of venous drainage in rat flaps: an experimental study. J Reconstr Microsurg 1989;5 (01):19-30

14 Miles DA, Crosby NL, Clapson JB. The role of the venous system in the abdominal flap of the rat. Plast Reconstr Surg 1997;99(07): 2030-2033

15 Suh H, Lee AY, Park EJ, Hong JP. Negative pressure wound therapy on closed surgical wounds with dead space: animal study using a swine model. Ann Plast Surg 2016;76(06):717-722

16 Almond LM, Khodaverdi L, Kumar B, Coveney EC. Flap anchoring following primary breast cancer surgery facilitates early hospital discharge and reduces costs. Breast Care (Basel) 2010;5(02): 97-101

17 Peter Suh HS, Hong JP. Effects of incisional negative-pressure wound therapy on primary closed defects after superficial circumflex iliac artery perforator flap harvest: randomized controlled study. Plast Reconstr Surg 2016;138(06):1333-1340

18 Kottayasamy Seenivasagam R, Gupta V, Singh G. Prevention of seroma formation after axillary dissection-a comparative randomized clinical trial of three methods. Breast J 2013;19(05): 478-484

19 Seretis K, Goulis D, Demiri EC, Lykoudis EG. Prevention of seroma formation following abdominoplasty: a systematic review and meta-analysis. Aesthet Surg J 2017;37(03):316-323

20 Kuc A, Dayicioglu D. Reducing wound tension with DuoDERM ${ }^{\mathrm{TM}}$ taping technique. Eplasty 2017;17:ic5

21 Cheng LF, Lee JT, Chou TD, et al. Experience with elastic rubber bands for the tie-over dressing in skin graft. Burns 2006;32(02): 212-215

22 Boyd JB, Carlisle IR, Thomson HG, Zuker RM. A rapid and effective method of skin graft stabilization in burned children. Ann Plast Surg 1982;9(05):400-401 